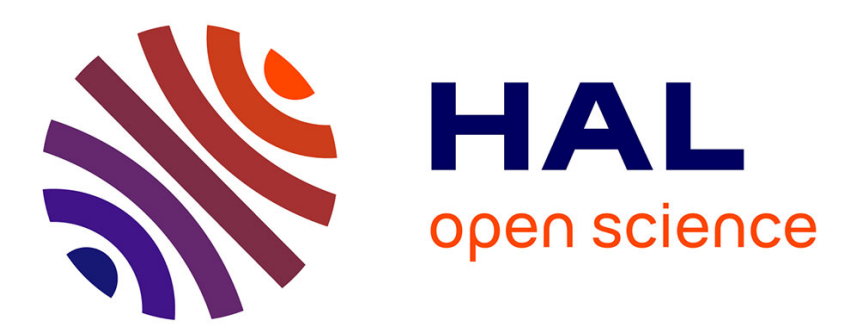

\title{
Applied geminography - symmetry analysis of twinned crystals and definition of twinning by reticular polyholohedry
}

\author{
Massimo Nespolo, Giovanni Ferraris
}

\section{- To cite this version:}

Massimo Nespolo, Giovanni Ferraris. Applied geminography - symmetry analysis of twinned crystals and definition of twinning by reticular polyholohedry. Acta Crystallographica Section A : Foundations and Advances [2014-..], 2004, 60, pp.89-95. 10.1107/S0108767303025625 . hal-00130548

\author{
HAL Id: hal-00130548 \\ https://hal.science/hal-00130548
}

Submitted on 12 Feb 2007

HAL is a multi-disciplinary open access archive for the deposit and dissemination of scientific research documents, whether they are published or not. The documents may come from teaching and research institutions in France or abroad, or from public or private research centers.
L'archive ouverte pluridisciplinaire HAL, est destinée au dépôt et à la diffusion de documents scientifiques de niveau recherche, publiés ou non, émanant des établissements d'enseignement et de recherche français ou étrangers, des laboratoires publics ou privés. 
Acta Crystallographica Section A

Foundations of

Crystallography

ISSN 0108-7673

Editor: D. Schwarzenbach

\section{Applied geminography - symmetry analysis of twinned crystals and definition of twinning by reticular polyholohedry}

\section{Massimo Nespolo and Giovanni Ferraris}


Acta Crystallographica Section A

Foundations of Crystallography

ISSN 0108-7673

Received 30 June 2003

Accepted 5 November 2003

C 2004 International Union of Crystallography

Printed in Great Britain - all rights reserved

\section{Applied geminography - symmetry analysis of twinned crystals and definition of twinning by reticular polyholohedry}

\author{
Massimo Nespolo ${ }^{\mathrm{a} *}$ and Giovanni Ferraris ${ }^{\mathrm{b}}$ \\ a Université Henri Poincaré Nancy 1, Laboratoire de Cristallographie et de Modélisation des \\ Matériaux Minéraux et Biologiques $\left(\mathrm{LCM}^{3} \mathrm{~B}\right)$, UMR - CNRS 7036, Faculté de Sciences et \\ Techniques, Boulevard des Aiguillettes, BP 239, F-54506 Vandœuvre-lès-Nancy CEDEX, France, \\ and ${ }^{\mathbf{b}}$ Università di Torino, Dipartimento di Scienze Mineralogiche e Petrologiche, Istituto di \\ Geoscienze e Georisorse, CNR, Via Valperga Caluso 35, 10125 Torino, Italy. Correspondence \\ e-mail: massimo.nespolo@Icm3b.uhp-nancy.fr
}

\begin{abstract}
The common classification of twinning into the four categories of twinning by merohedry (complete and exact overlap of the lattices of the twinned crystals), pseudomerohedry (complete but approximate overlap), reticular merohedry (partial but exact overlap) and reticular pseudomerohedry (partial and approximate overlap) is revised in terms of the complete (translational and point) lattice symmetry of the twin and of the individual. The new category of reticular polyholohedry is introduced for twins where the twin lattice has the same point symmetry but a different orientation of the individual lattice. It is shown that the degeneration to twin index 1 relates, in a parallel way, reticular merohedry to metric merohedry and reticular polyholohedry to syngonic merohedry. Some examples from the recent literature are analysed in terms of this revised classification.
\end{abstract}

\section{Introduction}

Twinned crystals are often considered an obstacle to the solution and refinement of crystal structures, and the tendency towards entrusting their treatment to black-box software packages is increasing. Besides the risks connected to an uncritical acceptance of the solution provided by the machine, one of the consequences of this trend is the increasing unawareness by some users of the symmetry and lattice peculiarities of twins, without which a real comprehension of the phenomenon of twinning is impossible.

Twins are such particular 'crystallographic objects' that J. D. H. Donnay called geminography the branch of crystallography dealing with twinning (Nespolo \& Ferraris, 2003). A twin is an oriented association of individual crystals of the same chemical and crystallographic species. The individuals in a twin are related by one or more geometrical laws (the twin laws) expressed through the point symmetry of the twin versus the point symmetry of the individual. The twin element (twin centre, twin axis, twin plane) is the geometric element about which twin operations (operations relating different individuals) are performed. ${ }^{1}$

\footnotetext{
${ }^{1}$ Twins are heterogeneous edifices formed by two or more homogeneous structurally three-periodic individuals (modules) related by a point-group operation. Consequently, the twin itself does not possess a homogeneous crystal structure. Homogeneous edifices built by structurally less-than-threeperiodic modules are termed cell twins: the cell-twin operation may have a translational component (Takéuchi, 1997; Ferraris et al., 2004).
}

The complete geminographical analysis of a twinned crystal should take into account three aspects:

1. the symmetry relations in direct space relating the individuals, the individual lattice and the twin lattice;

2 . the symmetry relations in reciprocal space and the effect of twinning on the diffraction pattern (Buerger, 1954, later republished in Buerger, 1962; Ferraris et al., 2004);

3. the morphology of the twin, as it appears in its forms (see, for example, Shafranovskii, 1973).

In this article, we deal with the first aspect, extending some of the classical categories introduced long ago and described in standard textbooks of crystallography.

Donnay \& Donnay (1974) classified twinning in two categories: TLS (twin lattice symmetry) and TLQS (twin lattice quasisymmetry) on the basis of the value of the obliquity $\omega$, which is the angle measuring the metrical symmetry deviation of the crystal lattice with respect to the twin lattice. A plane that is a (pseudo)symmetry plane for the lattice has a lattice row (quasi)perpendicular, which is a (pseudo)symmetry axis for the lattice. The obliquity $\omega$ is the complement to $90^{\circ}$ of the angle between these (pseudo)symmetry axis and plane; it is zero for TLS and non-zero for TLQS. In the diffraction pattern, TLS and TLQS differ by the absence or presence of split reflections, respectively (see Koch, 1992).

Well before, the so-called 'French school' (see Friedel, 1904, 1926) had introduced a finer classification into the four cate- 
gories of twinning by merohedry (complete and exact overlap of the lattices of the twinned crystals), pseudomerohedry (complete but approximate overlap), reticular merohedry (partial but exact overlap) and reticular pseudomerohedry (partial and approximate overlap). An alternative classification, based on the morphological - rather than reticular (pseudo)symmetry elements was introduced by the so-called 'German school' (see Hahn \& Klapper, 2003).

Buerger (1945) also discussed the influence of the structure on twinning and reinforced the concept of necessary conditions for twinning by adding the occurrence of structure (pseudo)symmetry besides that of reticular (pseudo)symmetry. Actually, a possible existence of 'sufficient' conditions for twinning appears contradicted by at least one fact: in the same batch of crystallization, the same compound may give at the same time twinned and untwinned crystals. The structural conditions added by Buerger for the occurrence of a given twin are thus themselves necessary but this necessity has a wider meaning than Friedel's reticular conditions. However, whereas the reticular conditions can be foreseen in a very general and simple way from the metric of the lattice, the structure must be already known in order to determine the structural conditions. In this paper, we apply Friedel's lattice treatment and introduce some generalizations that depend on the metric of the lattice only.

\section{Symmetry classification of twinning in terms of the lattice point group and translation group}

\subsection{Definitions}

We call 'individual' an untwinned crystal and denote by the subscript I all the related variables. The corresponding quantities for a twin are indicated by the subscript $\mathrm{T}$. The vector lattices of the individual and of the twin are thus $\mathbf{L}_{\mathrm{I}}$ and $\mathbf{L}_{\mathrm{T}}$.

The twin index $n_{\mathrm{T}}$ is the ratio of the number of lattice nodes of the individual to the number of nodes restored, exactly or approximately, by the twin operation (Friedel, 1904, 1926). It can be easily computed once the cells of $\mathbf{L}_{T}$ and $\mathbf{L}_{\mathrm{I}}$ are known. Let $m_{\mathrm{T}}$ and $m_{\mathrm{I}}$ be the multiplicities of the unit cells of $\mathbf{L}_{\mathrm{T}}$ and $\mathbf{L}_{\mathrm{I}}$, respectively; the value of $m$ is 1 for $P$ and $R, 2$ for $S$ (one face centred) and $I, 4$ for $F$ lattices. Let $\mathrm{O}\left(\mathcal{T}_{\mathrm{T}}\right)$ and $\mathrm{O}\left(\mathcal{T}_{\mathrm{I}}\right)$ be the (infinite) order of the group of translation [the group formed by the translation vectors of the lattice (Wondratschek, 2002)] of the twin $\left(\mathcal{T}_{\mathrm{T}}\right)$ and of the individual $\left(\mathcal{T}_{\mathrm{I}}\right)$. Let $V_{\mathrm{T}}$ and $V_{\mathrm{I}}$ be the volumes of the unit cells of $\mathbf{L}_{\mathrm{T}}$ and $\mathbf{L}_{\mathrm{I}}$, respectively. Let $v_{T}$ be the number of nodes of $\mathbf{L}_{\mathrm{I}}$ in the cell of $\mathbf{L}_{\mathrm{T}}$. Then the following equivalences hold:

$$
n_{\mathrm{T}}=\frac{O\left(\mathcal{T}_{\mathrm{I}}\right)}{O\left(T_{\mathrm{T}}\right)} \frac{m_{\mathrm{I}}}{m_{\mathrm{T}}}=\frac{V_{\mathrm{T}}}{V_{\mathrm{I}}} \frac{m_{\mathrm{I}}}{m_{\mathrm{T}}}=\frac{v_{\mathrm{T}}}{m_{\mathrm{T}}} .
$$

Let $\mathcal{H}$ be the (vector) point group of the individual, and $\mathcal{D}$ the holohedral supergroup of $\mathcal{H}$ : if $\mathcal{H}$ is already holohedral, then $\mathcal{D}=\mathcal{H}$. The point symmetry of $\mathbf{L}, \mathcal{D}(\mathbf{L})$, is always holohedral. Normally, $\mathcal{D}=\mathcal{D}(\mathbf{L})$, but when the crystal has accidentally a specialized higher metric, then $\mathcal{D}(\mathbf{L})$ is a supergroup of $\mathcal{D}$ :

$$
\begin{array}{ll}
\text { non-specialized metric: } & \mathcal{D}(\mathbf{L})=\mathcal{D} \supseteq \mathcal{H} \\
\text { specialized higher metric: } & \mathcal{D}(\mathbf{L}) \supset \mathcal{D} \supseteq \mathcal{H} .
\end{array}
$$

$\mathcal{D}(\mathbf{L})$ and $\mathcal{D}$ can be decomposed in cosets ( $c f$. Giacovazzo, 2002a)

$$
\begin{aligned}
\mathcal{D}(\mathbf{L}) & =\mathcal{D} \cup \mathcal{C}_{1}^{\mathcal{D}} \cup \mathcal{C}_{2}^{\mathcal{D}} \cup \ldots \cup \mathcal{C}_{n-1}^{\mathcal{D}} \\
\mathcal{D} & =\mathcal{H} \cup \mathcal{C}_{1}^{\mathcal{H}} \cup \mathcal{C}_{2}^{\mathcal{H}} \cup \ldots \cup \mathcal{C}_{n^{\prime}-1}^{\mathcal{H}}
\end{aligned}
$$

where $\mathcal{C}_{j}^{\mathcal{D}}$ and $\mathcal{C}_{j}^{\mathcal{H}}$ are the cosets, $n$ and $n^{\prime}$ the indices of $\mathcal{D}(\mathbf{L})$ in $\mathcal{D}$ and of $\mathcal{D}$ in $\mathcal{H}$, respectively. Without an accidentally specialized metric, $n=1$ and $(3 a)$ reduces to $(2 a)$. When $\mathcal{H}$ is holohedral, $n^{\prime}=1$.

Friedel's geometrical crystallography was based on the lattice symmetry, but his classification of twinning took into account only the point symmetry of lattices. To describe some special cases in the general framework of Friedel's classification (Friedel, 1904, 1926), we need to consider also the translational symmetry.

The coordinate systems of the individual and of the twin are related by a transformation matrix $\mathbf{P}$ that implies, in general, a change of the basis vectors:

$$
\left\langle\left.\mathbf{a b c}\right|_{\mathrm{I}} \mathbf{P}=\left\langle\left.\mathbf{a b c}\right|_{\mathrm{T}} .\right.\right.
$$

$\mathbf{P}$ can be decomposed into the product of a rotation $\mathbf{R}_{\mathbf{u}}(\varphi)$ by $\varphi$ around a rational direction $\mathbf{u}$ in $\left\langle\left.\mathbf{a b c}\right|_{\mathrm{I}}\right.$ and a metric expansion $\mathbf{X}$ (lattice thinning):

$$
\mathbf{P}=\mathbf{R}_{\mathbf{u}}(\varphi) \mathbf{X}
$$

Let us indicate by $\mathbf{W}$ the matrix representation of a symmetry operation for $\mathbf{L}_{\mathrm{I}}$, and by $\mathbf{W}_{\mathrm{T}}$ the matrix representing the twin operation. TLS twinning can thus be classified as follows.

2.1.1. $\mathcal{T}_{\mathrm{T}} \subset \mathcal{T}_{\mathrm{I}}$ and $\mathcal{D}(\mathbf{L})_{\mathrm{T}} \neq \mathcal{D}(\mathbf{L})_{\mathrm{I}}$. This case corresponds to reticular merohedry in Friedel's $(1904,1926)$ classification. Actually, Friedel (1926, p. 30) restricted this category to the case in which the unit cell of $\mathbf{L}_{\mathrm{T}}$ has a symmetry higher than that of $\mathbf{L}_{\mathrm{I}}\left[\mathcal{T}_{\mathrm{T}} \subset \mathcal{T}_{\mathrm{I}}\right.$ and $\left.\mathcal{D}(\mathbf{L})_{\mathrm{T}} \supset \mathcal{D}(\mathbf{L})_{\mathrm{I}}\right]$. Friedel, indeed, discussed cases of reticular merohedry in which $\mathcal{D}(\mathbf{L})_{\mathrm{T}} \subset \mathcal{D}(\mathbf{L})$ (holohedral point group of the twin lower than the holohedral point group of the individual), for example in the case of twins of $\beta$-quartz (Friedel, 1923), but in his classification he apparently overlooked these cases. The condition $\mathcal{T}_{\mathrm{T}} \subset \mathcal{T}_{\mathrm{I}}$ and $\mathcal{D}(\mathbf{L})_{\mathrm{T}} \supset \mathcal{D}(\mathbf{L})_{\mathrm{I}}$ is verified in the case of twins with parallel axes, but not necessarily for twins with inclined axes. In the latter case, in fact, the twin point group is a supergroup no longer of the point group of the individual but of the intersection group of the individuals in their respective orientations (Nespolo, 2004).

Hahn \& Klapper (2003) chose to extend the definition of reticular merohedry in terms of the $\mathcal{T}_{\mathrm{T}} \subset \mathcal{T}_{\mathrm{I}}$ relation only, speaking thus of " "merohedry' of translation groups (lattices)". We substantially agree with the concept of 'merohedry of translation groups', but recommend treating separately the special case of identical point groups of lattices, as discussed in $\$ 2.1 .2$.

Twinning by reticular merohedry can be classified on the basis of $\mathbf{R}$ and $\mathbf{X}$ in equation (5). 
(i) $\mathbf{R}_{\mathbf{u}}(\varphi) \neq \mathbf{W}, \mathbf{X} \neq \mathbf{I}$ ( $n$ integer $>1$; $\mathbf{I}$ is the identity matrix). $\mathbf{L}_{\mathrm{I}}$ and $\mathbf{L}_{\mathrm{T}}$ have different orientation and different metric.

(ii) $\mathbf{R}_{\mathbf{u}}(\varphi)=\mathbf{W}, \mathbf{X} \neq n \mathbf{I}(n$ integer $>1)$. $\mathbf{L}_{\mathbf{I}}$ and $\mathbf{L}_{\mathrm{T}}$ have the same orientation but different metric.

$\mathcal{D}(\mathbf{L})_{\mathrm{T}}$ can be decomposed into cosets in terms of $\mathcal{D}(\mathbf{L})_{\mathrm{I}}$ :

$$
\mathcal{D}(\mathbf{L})_{\mathrm{T}}=\mathcal{D}(\mathbf{L})_{\mathrm{I}} \cup \mathcal{C}_{1}^{I} \cup \mathcal{C}_{2}^{I} \cup \ldots \cup \mathcal{C}_{n^{\prime \prime}-1}^{I} ;
$$

the twin operation belongs to one of the cosets: $\mathbf{W}_{\mathrm{T}} \in \mathcal{C}_{j}^{I}, j>0$. All the operations in one coset are equivalent under the action of the symmetry operations of $\mathcal{D}(\mathbf{L})_{\text {I }}$ and any of them can be taken as representative of the coset. When one of these operations uniquely explains the morphology developed by the twin, it is known as representative (twin) operation of the coset (Nespolo \& Ferraris, 2000).

Example. Rhombohedral crystals, as well as cubic crystals with $I$ - or $F$-centred cells, often show the so-called 'obverse/ reverse' twinning. A lattice of type $h R, c I$ or $c F$ has an $h P$ sublattice, and the symmetry elements of this sublattice, which are not symmetry elements for the whole lattice, may act as twin elements. The term 'obverse/reverse twinning' must be discouraged in our opinion. It comes from the fact that as an independent twin operation one can always take the twofold component of the sixfold axis for the twin lattice: applied to the rhombohedral lattice (or the rhombohedral component of the $c I$ or $c F$ lattice), it corresponds to changing the rhombohedral cell from the obverse setting to the reverse setting, or vice versa. This twinning is often described as a 'special kind of twinning' but it is actually a classical twinning by reticular merohedry: the only 'special' feature is that for an $h R$ lattice there always exists a corresponding $h P$ sublattice. In terms of lattice and point symmetry, it corresponds to: $\mathcal{T}_{\mathrm{T}} \subset \mathcal{T}_{\mathrm{I}}$, $\mathcal{D}(\mathbf{L})_{\mathrm{T}}=6 / \mathrm{mmm} \supset \mathcal{D}(\mathbf{L})_{\mathrm{I}}=\overline{3} \mathrm{~m}$.

2.1.2. $\mathcal{T}_{\mathrm{T}} \subset \mathcal{T}_{\mathrm{I}}$ but $\mathcal{D}(\mathbf{L})_{\mathrm{T}}=\mathcal{D}(\mathbf{L})_{\mathrm{I}}$. This case corresponds to $\mathbf{R}_{\mathbf{u}}(\varphi) \neq \mathbf{W}, \mathbf{X}=n \mathbf{I}(n$ integer $>1)$. Each symmetry element for $\mathbf{L}_{\mathrm{T}}$ differently oriented from the corresponding elements in $\mathbf{L}_{\mathbf{I}}$ can act as twin element. As mentioned above, this case does not rigorously enter into Friedel's classification because there is no relation of merohedry between the $\mathbf{L}_{\mathrm{T}}$ and the $\mathbf{L}_{\mathrm{I}}$. For this reason, we introduce (Ferraris \& Nespolo, 2003; Ferraris et al., 2004) the term reticular polyholohedry ${ }^{2}$ to indicate the presence of the same holohedry in the lattice of the individual and in differently oriented sublattice(s). Of course, pseudopolyholohedry is possible as well; see the examples in $\S 3.5$.

2.1.3. $\mathcal{T}_{\mathrm{T}}=\mathcal{T}_{\mathrm{I}}$ and $\mathcal{D}(\mathbf{L})_{\mathrm{T}}=\mathcal{D}(\mathbf{L})_{\mathrm{I}}$. This case implies $\mathbf{R}_{\mathbf{u}}(\varphi)=\mathbf{W}, \mathbf{X}=\mathbf{I}$. $\mathbf{L}_{\mathbf{I}}$ and $\mathbf{L}_{\mathrm{T}}$ have the same orientation and the same metric, i.e. they coincide. Twinning can only be by merohedry, according to Friedel's classification. It is further subdivided depending on whether $\mathcal{D}(\mathbf{L})$ coincides with $\mathcal{D}$ or is a supergroup of it. Until recently (Nespolo \& Ferraris, 2000), the following three cases were not clearly recognized as distinct but treated comprehensively as 'merohedry'.

1. The lattice does not have an accidentally specialized higher metric and the twin operation belongs to one of the

\footnotetext{
${ }^{2}$ Evidently, reticular polyholohedry in triclinic crystals corresponds to the trivial case of the choice of a differently oriented multiple cell, without any new twin element, the only possible one being the inversion centre for hemihedral crystals.
}

cosets in equation $(3 b): \mathbf{W}_{\mathrm{T}} \in \mathcal{C}_{j}^{\mathcal{H}}, j>0$. In other words, the twin operation belongs to $\mathcal{D}$, the holohedral point symmetry of the crystal. This is the most common case of twinning by merohedry, which is now termed twinning by syngonic merohedry (Nespolo \& Ferraris, 2000).

2. The lattice has an accidentally specialized higher metric and the point group of the crystal is holohedral $(\mathcal{D}=\mathcal{H})$. $\mathcal{H}$ is however merohedral with respect to the point group of the lattice $[\mathcal{D}(\mathbf{L}) \supset \mathcal{D}=\mathcal{H}]$. The twin operation belongs to the point symmetry of the lattice, $\mathcal{D}(\mathbf{L})$, but not to the holohedral point symmetry of the crystal, $\mathcal{D}=\mathcal{H}: \mathbf{W}_{\mathrm{T}} \in \mathcal{C}_{j}^{\mathcal{D}}, j>0$ [equation $3 a)$ ]. This type of merohedry, and the corresponding twinning, is now termed metric merohedry ${ }^{3}$ (Nespolo \& Ferraris, 2000).

3. The lattice has an accidentally specialized higher metric but the point group of the crystal is not holohedral $(\mathcal{D} \supset \mathcal{H})$. The crystal is thus doubly merohedral: with respect to $\mathcal{D}$ (syngonic merohedry) and with respect to $\mathcal{D}(\mathbf{L})$ (metric merohedry). Depending on whether the twin operation corresponds to equation $(3 a)$ or $(3 b)$, twinning is by metric or by syngonic merohedry. The two types of twinning can coexist.

With respect to the effect of twinning on the diffraction intensities, merohedric twins are divided into class I (the twin operation is equivalent to an inversion centre and the intensities from the individuals are equivalent under Friedel's law), class IIA (twinning by syngonic merohedry with the twin law not equivalent to an inversion centre) and class IIB (twinning by metric merohedry) (Catti \& Ferraris, 1976; Nespolo \& Ferraris, 2000).

Example. A monoclinic holoaxial crystal $(\mathcal{H}=2)$ with $\beta=$ $90^{\circ}$ is syngonically merohedral with respect to the monoclinic holohedry $(\mathcal{D}=2 / \mathrm{m})$ but metrically merohedral with respect to its lattice $[\mathcal{D}(\mathbf{L})=\mathrm{mmm}]$. The individual may undergo twinning by syngonic merohedry with respect to $\mathcal{D}$, the twin point group being $2 / \mathrm{m}^{\prime}$ [about symbols of twin point groups, see Curien \& Le Corre (1958) and Nespolo (2004)]. It may however undergo twinning by metric merohedry with respect to $\mathcal{D}(\mathbf{L})$, the twin point group being either $2^{\prime} 22^{\prime}$ or $m^{\prime} m 2^{\prime}$. In this case, the monoclinic hemihedral individual is twinned with respect to the orthorhombic hemihedry but not to the monoclinic holohedry (point groups of the same order). It may finally undergo twinning with respect to the orthorhombic holohedry: in this case, both types of twinning are present simultaneously, because twinning by syngonic merohedry with respect to the monoclinic holohedry is part of the total twinning. As a result, two independent twin elements are active, which originate a four-individual twin, the twin point group being $2^{\prime \prime} / m^{\prime \prime} 2 / m^{\prime} 2^{\prime \prime} / m^{\prime \prime}$.

2.1.4. Generalized classification of twinning. On the basis of the above discussion, the correspondences in Table 1 are immediately established. The metric merohedry corresponds

\footnotetext{
${ }^{3}$ Friedel (1904, p. $143 ; 1926$, pp. 56-57) had essentially denied the possibility of the occurrence of metric merohedry, which he termed 'higher-order merohedry' (mériédrie d'ordre supérieur), as either unlikely or equivalent to a pseudomerohedry of low obliquity. Nowadays, several examples of true metric merohedry (within experimental error) are known, and after the first one officially reported (Ferraris et al., 2001), older examples have been reclassified in this category (see also $\$ 3.2$ ).
} 
Table 1

Symmetry classification of twinning.

\begin{tabular}{lllll}
\hline Type of twinning & $\begin{array}{l}\text { Lattice } \\
\text { relation }\end{array}$ & $\begin{array}{l}\text { Group of } \\
\text { translation }\end{array}$ & Point group & $\begin{array}{l}\text { Twin } \\
\text { index }\end{array}$ \\
\hline Reticular merohedry & $\mathbf{L}_{\mathrm{T}} \neq \mathbf{L}_{\mathrm{I}}$ & $\mathcal{T}_{\mathrm{T}} \subset \mathcal{T}_{\mathrm{I}}$ & $\mathcal{D}(\mathbf{L})_{\mathrm{T}} \neq \mathcal{D}(\mathbf{L})_{\mathrm{I}}$ & $n_{\mathrm{T}}>1$ \\
Reticular polyholohedry & $\mathbf{L}_{\mathrm{T}} \neq \mathbf{L}_{\mathrm{I}}$ & $\mathcal{T}_{\mathrm{T}} \subset \mathcal{T}_{\mathrm{I}}$ & $\mathcal{D}(\mathbf{L})_{\mathrm{T}}=\mathcal{D}(\mathbf{L})_{\mathrm{I}}$ & $n_{\mathrm{T}}>1$ \\
Metric merohedry & $\mathbf{L}_{\mathrm{T}}=\mathbf{L}_{\mathrm{I}}$ & $\mathcal{T}_{\mathrm{T}}=\mathcal{T}_{\mathrm{I}}$, & $\mathcal{D}(\mathbf{L})_{(\mathrm{T}=\mathrm{I})} \supset \mathcal{D}$ & $n_{\mathrm{T}}=1$ \\
Syngonic merohedry & $\mathbf{L}_{\mathrm{T}}=\mathbf{L}_{\mathrm{I}}$ & $\mathcal{T}_{\mathrm{T}}=\mathcal{T}_{\mathrm{I}}$, & $\mathcal{D}(\mathbf{L})_{(\mathrm{T}=\mathrm{I})}=\mathcal{D}$ & $n_{\mathrm{T}}=1$ \\
\hline
\end{tabular}

to the degeneration of reticular merohedry to $n_{\mathrm{T}}=1$ and $\mathcal{T}_{\mathrm{T}}=$ $\mathcal{T}_{\text {I }}$. In fact, because $\mathbf{L}_{\mathrm{T}}$ and $\mathbf{L}_{\mathrm{I}}$ coincide, the point groupsubgroup relation is transferred from $\mathcal{D}(\mathbf{L})_{\mathrm{T}}$ versus $\mathcal{D}(\mathbf{L})_{\mathrm{I}}$ in the case of reticular merohedry, and to $\mathcal{D}(\mathbf{L})$ versus $\mathcal{D}$ in the case of metric merohedry. In the same way, the syngonic merohedry corresponds to the degeneration of reticular polyholohedry to $n_{\mathrm{T}}=1$ and $\mathcal{T}_{\mathrm{T}}=\mathcal{T}_{\mathrm{I}}$. In fact, because $\mathbf{L}_{\mathrm{T}}$ and $\mathbf{L}_{\mathrm{I}}$ coincide, the point-group equivalence is transferred from $\mathcal{D}(\mathbf{L})_{\mathrm{T}}$ versus $\mathcal{D}(\mathbf{L})_{\mathrm{I}}$, in the case of reticular polyholohedry to $\mathcal{D}(\mathbf{L})$ versus $\mathcal{D}$ in the case of syngonic merohedry.

\subsection{The number of individuals}

The number of individuals in a twin is determined by the number of independent twin elements (i.e. twin elements that are not equivalent under the point symmetry of the individual) and by the order of each of these elements. The action of nonindependent twin elements does not produce further individuals: although obvious, this fact is sometimes overlooked (e.g. Takano, 1972). Because each individual is (formally) generated by the action of a point-group operation, each individual has a different orientation. One may object that in polysynthetic twins, which can be represented with the scheme $A B A B A B \ldots$ (for a binary case; extension to a higher number of individuals is trivial), this is no longer true. Polysynthetic twins actually consist of parallel repeated intergrowths and the individuals with the same orientation are simply parallel growth alternated with individuals in twin orientation. They can also be interpreted as repeated application of the same twin operation.

If $n_{E}$ is the number of independent twin elements (twin degree: Nespolo, 2004), $O_{j}^{T}$ the global order of the $j$ th twin element, and $O_{j}^{S}$ the order of the same element as symmetry element for the individuals, the number of individuals (with different orientation, as explained above) $n_{\mathrm{I}}$ is:

$$
n_{I}=\prod_{j=1}^{n_{E}} \frac{O_{j}^{T}}{O_{j}^{S}} .
$$

Obviously, $O_{j}^{S}=1$ for binary and ternary twin elements, whereas $O_{j}^{S} \geq 1$ for quaternary and senary twin elements, depending on whether a component ( 2 of $4 ; 2$ or 3 of 6 ) of the same element acts as symmetry element (for the individual: merohedry; for the individual lattice: reticular merohedry) or not. For example, a monoclinic crystal with a hexagonal sublattice may give a second-degree twin (two independent twin elements) where the first independent twin element is a sixfold axis parallel to the twofold monoclinic axis $\left(O_{1}^{T} / O_{1}^{S}=6 / 2=3\right)$ and the second independent twin element is a twofold axis perpendicular to the sixfold axis $\left(O_{2}^{T} / O_{2}^{S}=2 / 1=2\right)$. The number of individuals is thus $\left(O_{1}^{T} / O_{1}^{S}\right)\left(O_{2}^{T} / O_{2}^{S}\right)=3 \times 2=6$.

\subsection{Peculiarities of the twin lattice quasisymmetry (TLQS)}

In the case of TLS, the twin lattice is defined by the subset of nodes corresponding to the exact superposition of the lattices of the individuals. When the obliquity is non-zero, this definition no longer applies rigorously because the superposition is only approximated. The twin lattice in this case is uniquely defined by the action of the $\mathcal{D}(\mathbf{L})_{\mathrm{T}}$ point group on the lattice of any of the individuals, and corresponds to the degeneration to zero obliquity of the imperfect superposition of the individual lattices. One of the consequences is the well known fact that corresponding twin rotation and reflection operations in centrosymmetric crystals, which are equivalent in the case of TLS, are no longer equivalent in the case of TLQS. They are called 'reciprocal twins' (Mügge, 1898) or 'corresponding twins' (Friedel, 1904, 1926).

TLQS twinning is subdivided into reticular pseudomerohedry (twin index $\left.n_{\mathrm{T}}>1\right)$ and pseudomerohedry $\left(n_{\mathrm{T}}=1\right)$. The degeneration of reticular pseudomerohedry to $\omega=0$ corresponds quite obviously either to the reticular merohedry, if $\mathcal{D}(\mathbf{L})_{\mathrm{T}} \neq \mathcal{D}(\mathbf{L})_{\mathrm{I}}$, or to the reticular polyholohedry, if $\mathcal{D}(\mathbf{L})_{\mathrm{T}}=$ $\mathcal{D}(\mathbf{L})_{\text {I }}$, whereas the degeneration of pseudomerohedry to $\omega=0$ corresponds to the metric merohedry.

Very low values of $\omega$ make it difficult to distinguish between pseudomerohedry and metric merohedry, and between reticular pseudomerohedry and reticular merohedry.

\section{Geminographical analysis of examples from the literature}

Multiple twinning - where more than two individuals are related by the twin element(s) - pseudosymmetries and accidental lattice metric symmetries are often described without reference to the classical (Friedel, Donnay) nomenclature discussed in this paper. In this section, we revisit some examples of discordant nomenclature that have recently appeared in the literature. A systematic analysis, which is outside the scope of this article, would certainly reveal several other examples.

\subsection{Twinning by pseudomerohedry}

Twinning in the technologically important perovskites is particularly troublesome because of the lowering of the ideal cubic symmetry that often occurs (cf. Ferraris et al., 2004). Among several others, we refer to the paper by Arakcheeva et al. (1997) that describes twinning in natural orthorhombic perovskite $\mathrm{CaTiO}_{3}(a=5.378, b=5.444, c=7.637 \AA$ А, Pbnm $)$ and also take the opportunity of correcting some basic mistakes. Twinning is by reticular pseudomerohedry, twin index 2, the twin lattice being pseudocubic (the covariant transformation matrix is $011 / 0 \overline{1} 1 / 100)$. The authors identified four individuals but incorrectly described the twin laws. In fact, they gave two twin laws, $\{112\}$ and $\{010\}$ (orthorhombic 
reference); all planes correspond to diagonal planes $\{110\}$ in the cubic sublattice. However, the (010) plane is a symmetry plane for the individual, which is holohedral: this plane cannot thus act as twin plane. The other two planes the authors apply

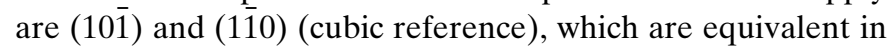
the crystal because they belong to the $\{112\}$ form, and produce thus only a first pair of individuals. The authors mention 'conjugate twins' (a further non-standard expression for corresponding twins), which may explain the second pair of individuals (assuming these were really present). This pair was most probably obtained by the action of the $\langle 112\rangle$ twin axis, which corresponds to the $\langle 110\rangle$ twofold axis of the cubic lattice: twin axis and twin plane are not mutually perpendicular in the individual lattice and thus their effects are different even if the individual is centrosymmetric.

\subsection{Twinning by metric merohedry}

3.2.1. $\mathbf{C}_{19} \mathbf{H}_{33} \mathbf{L i N}_{3}$ Tl. Herbst-Irmer \& Sheldrick (1998) interpreted as 'twin by pseudomerohedry' the twin found in monoclinic $P 2_{1} / c, \mathrm{C}_{19} \mathrm{H}_{33} \mathrm{LiN}_{3} \mathrm{Tl}(a=13.390, b=25.604, c=$ $13.390 \AA, \beta=112.39^{\circ}$ ). These authors, followed by Giacovazzo (2002b), noted that the $m P$ lattice admits an $o S$ cell $(a=$ $14.900, b=22.252, c=25.604 \AA)$ and interpreted the twin as originated by $\{010\}$ twinning (orthorhombic reference). On the basis of the analysis presented above, this example should be more properly described as follows. The structure is monoclinic, but its lattice is orthorhombic: the $o S$ cell is in fact nothing else than the conventional cell of the orthorhombic lattice, of which the $m P$ cell represents the primitive cell (note $a=c)$. The twin element is the (010) plane [(101) in the $m P$ reference], which belongs to the lattice of the individual. It is thus obvious that $\mathcal{T}_{\mathrm{T}}=\mathcal{T}_{\mathrm{I}}, \mathcal{D}(\mathbf{L})_{(\mathrm{T} \equiv \mathrm{I})}=m m m, \mathcal{D}=\mathcal{H}=2 / m$ : twinning is by metric merohedry. The same unrecognized type of twin occurs in the opioid peptide reported by FlippenAnderson et al. (2001) and in cafetite (Krivovichev et al., 2003). The latter case is quite subtle because the metrically orthorhombic $C$-centred cell $(a=4.944, b=31.435, c=$ $12.109 \AA$ ), which is obtained from the monoclinic one published by the authors $(a=4.944, b=12.109, c=15.911 \AA$, $\left.\beta=98.94^{\circ} ; P 2_{1} / n\right)$ via the transformation $\overline{1} 00 / \overline{1} 0 \overline{2} / 010$, has a very obtuse primitive monoclinic cell $(a=c=15.91, b=$ $12.109 \AA, \beta=162.13^{\circ}$ ). Unfortunately, the usual crystallographic software that explores for sub- and supercells ignores such obtuse cells.

3.2.2. ( $\eta^{8}$-Cyclooctatetraenyl)[hydrotris(pyrazolyl)borato]titan(III). The twinning in the title compound, presented by Herbst-Irmer \& Sheldrick (1998) as example 2 of twinning by pseudomerohedry, is actually by metric merohedry. In fact, the $m C$ cell $\left(a=10.220, b=11.083, c=7.538 \AA, \beta=96.85^{\circ}, C m\right)$ has a metrically $h R$ primitive cell with $a=7.538 \AA$ and $\alpha=94.64^{\circ}$, and the twin element is $3_{[111]}$ (rhombohedral indexing). Thus, $\mathcal{T}_{\mathrm{T}}=\mathcal{T}_{\mathrm{I}}, \mathcal{D}(\mathbf{L})_{(\mathrm{T} \equiv \mathrm{I})}=\overline{3} m \supset \mathcal{D}=2 / m \supset \mathcal{H}=m$.

\subsection{Twinning by reticular merohedry}

3.3.1. $\mathrm{NaBa}_{2} \boldsymbol{M}_{2}^{2+} \mathbf{M}^{3+} \mathrm{O}_{6}(\boldsymbol{M}=\mathrm{Ni}, \mathrm{Cu})$. Quarez et al. (2002) described twinning in $\mathrm{NaBa}_{2} M_{2}^{2+} M^{3+} \mathrm{O}_{6}(M=\mathrm{Ni}, \mathrm{Cu})$ as 'polysynthetic twinning' with a 'misleading $C$-centred orthorhombic false symmetry'. The crystal is orthorhombic, spacegroup type $F m m m$, with a perfectly hexagonal mesh in the (001) plane $(a=8.296, b=14.369, c=11.225 \AA$ for $M=\mathrm{Ni} ; a=$ 8.416, $b=14.577, c=11.418 \AA$ for $M=\mathrm{Cu} ; b=a 3^{1 / 2}$ in both cases). Based on this hexagonal mesh $(a=8.296 \AA)$, an $h P$ sublattice exists that corresponds to the twin lattice. The threefold component of the sixfold axis of this sublattice acts as twin element: $\mathcal{T}_{\mathrm{T}} \subset \mathcal{T}_{\mathrm{I}}, \mathcal{D}(\mathbf{L})_{\mathrm{T}}=6 / \mathrm{mmm} \supset \mathcal{D}(\mathbf{L})_{\mathrm{I}}=\mathcal{D}=\mathcal{H}=$ $\mathrm{mmm}$ : twinning is by reticular merohedry, twin index 2 .

The twin is not at all 'polysynthetic', but simply a threeindividual twin. The $C$-centred orthorhombic symmetry is neither 'misleading' nor 'false': it simply corresponds to one of the three orthohexagonal cells of the hexagonal sublattice $\left(a_{o}=a_{h}, b_{o}=2 b_{h}+a_{h}\right.$ or equivalent transformation; $b_{o}=$ $\left.a_{o} 3^{1 / 2}\right)$.

3.3.2. Temperature-dependent twinning in a fluorocyclohexane/thiourea inclusion compound. The low-temperature phase of a fluorocyclohexane/thiourea inclusion compound (Yeo et al., 2001) is monoclinic with orthorhombic metric symmetry $\left(a=27.52, b=15.718, c=12.33 \AA, \beta=90^{\circ}, P 2_{1} / n\right)$ and the high-temperature phase is rhombohedral $R \overline{3} c(a=$ 15.971, $c=12.495 \AA$, hexagonal axes). Both phases show twinning, which, for the rhombohedral phase, has been interpreted by using the 'obverse/reverse' formalism discussed in \$2.1.1: $\mathcal{T}_{\mathrm{T}} \subset \mathcal{T}_{\mathrm{I}}, \mathcal{D}(\mathbf{L})_{\mathrm{T}}=6 / \mathrm{mmm} \supset \mathcal{D}(\mathbf{L})_{\mathrm{I}}=\overline{3} m$. The twinning of the low-temperature phase is by metric merohedry: $\mathcal{T}_{\mathrm{T}} \equiv$ $\mathcal{T}_{\mathrm{I}}, \mathcal{D}(\mathbf{L})_{\mathrm{T} \equiv \mathrm{I}}=m m m \supset \mathcal{D}_{\mathrm{I}}=\mathcal{H}=2 / m$. However, the twin law has been introduced only via analogy with the high-temperature phase twinning.

\subsection{Combined twinning}

The multiple twinning observed in the hexagonal-related perovskite $\mathrm{S}_{3}\left(\mathrm{Ru}_{0.336} \mathrm{Pt}_{0.664}\right) \mathrm{CuO}_{6}$ (Friese et al., 2003) has been explained by 'a combination of rhombohedral obverse/reverse twinning plus the threefold axis from the trigonal system'. The twin matrices shown in Table 2 therein simply correspond to the $\left(6_{[001]}\right)^{n}(1 \leq n \leq 5)$ symmetry operations of the $h P$ lattice. As a matter of fact, the conventional $m C$ cell $(a=9.294$, $b=9.595, c=6.679 \AA, \beta=92.63^{\circ}$ ), given by the authors in Appendix $A$, is metrically rhombohedral (transformation $110 / \overline{1} 10 / 001$ ), with $a=6.679 \AA$ and $\alpha=91.83^{\circ}$. The authors refined the structure in the unusual space-group setting $R 12 / c$, $a=b=9.595, c=11.193 \AA, \gamma=120^{\circ}$. Therefore, they implicitly recognized that their compound belongs to the rhombohedral lattice system but to the monoclinic crystal system. Actually, this is an example of a twin that combines two types of twinning: metric merohedry and reticular merohedry. The lattice of the individual has an accidentally specialized metric, namely rhombohedral. Thus, $\mathcal{T}_{\mathrm{T}}=\mathcal{T}_{\mathrm{I}}, \mathcal{D}(\mathbf{L})=\overline{3} m \supset \mathcal{D}=\mathcal{H}=$ $2 / m$. The threefold axis corresponds to a symmetry axis for $\mathcal{D}(\mathbf{L})$ but not for $\mathcal{D}$. The other twin element corresponds to reticular merohedry, $n_{\mathrm{T}}=3$. In fact, the twin lattice is $h P$, which is a sublattice of $h R: \mathcal{T}_{\mathrm{T}} \subset \mathcal{T}_{\mathrm{I}}, \mathcal{D}(\mathbf{L})_{\mathrm{T}}=6 / \mathrm{mmm} \supset$ $\mathcal{D}(\mathbf{L})_{\mathrm{I}}=\overline{3} m$. 
In the closely related $\mathrm{Sr}_{3} \mathrm{CuPtO}_{6}(a=9.317, b=9.720, c=$ $6.685 \AA, \beta=91.95^{\circ}, C 2 / c$; Hodeau et al., 1992), the primitive rhombohedral cell was recognized even if the observed twinning was not strictly discussed in terms of its metric merohedry.

\subsection{Twinning by reticular (pseudo)polyholohedry}

3.5.1. Melilite. Bindi et al. (2003) have reported a case of twinning in melilite that presents peculiar characteristics. The diffraction data of a melilite $\left(\mathrm{Ca}_{1.70} \mathrm{Sr}_{0.07} \mathrm{Na}_{0.20} \mathrm{~K}_{0.03}\right)$ $\left(\mathrm{Mg}_{0.70} \mathrm{Fe}_{0.04}^{2+} \mathrm{Al}_{0.25} \mathrm{Fe}_{0.01}^{3+}\right)\left(\mathrm{Si}_{1.92} \mathrm{Al}_{0.08}\right) \mathrm{O}_{7}$ collected by an areadetector single-crystal diffractometer could be indexed only on the basis of a tetragonal supercell with $a_{s}=5^{1 / 2} a_{b}, c_{s}=c_{b}$, which is five times larger than the basic cell of melilite, $a_{b}=$ $7.775, c_{b}=5.032 \AA$. The supercell is oriented such that its $(100)$ symmetry plane corresponds to the (12̄0) plane of the basic cell and acts as twin plane. The authors, on the basis of the non-space-group absences and of the known basic cell, were able to interpret the diffraction pattern of the twin and classified it as a 'non-merohedral' twin (twin index 5), an ambiguous expression incorrectly used instead of 'non-merohedric' to indicate a twin by reticular (pseudo)merohedry (Nespolo \& Ferraris, 2003).

In this case, both the twin lattice and the individual lattice are tetragonal, so that $\mathcal{T}_{\mathrm{T}} \subset \mathcal{T}_{\mathrm{I}}, \mathcal{D}(\mathbf{L})_{\mathrm{T}}=\mathcal{D}(\mathbf{L})_{\mathrm{I}}=4 / \mathrm{mmm}$, $n_{\mathrm{T}}=5$. Therefore, this twin is by reticular polyholohedry, rather than by reticular merohedry. Another similar example occurs in $\mathrm{SmS}_{1.9}$ (Tamazyan et al., 2000).

3.5.2. Jonesite. Jonesite (Krivovichev \& Armbruster, 2004) is monoclinic $\left(a=8.694, b=25.918, c=8.694 \AA\right.$, $\beta=104.73^{\circ}$, $P 2_{1} / m$ ). Because $a=c$, the lattice of jonesite is metrically orthorhombic with a conventional $C$-centred cell whose parameters are $a=10.618, b=13.770, c=25.918 \AA$. Jonesite shows twinning by metric merohedry: $\mathcal{D}(\mathbf{L})=m m m \supset \mathcal{D}=\mathcal{H}=$ $2 / m, \mathcal{C}_{j}^{\mathcal{D}}, j \geq 0$ [equation (3a)]. Moreover, by the transformation $\overline{100 / 010 / 104}$, a pseudo-orthorhombic subcell (obliquity $\left.0.25^{\circ} ; \quad a=8.694, b=25.918, c=33.633 \AA, \beta=89.75^{\circ}\right)$ is obtained. The symmetry elements of this cell are differently oriented relative to those of the orthorhombic metric symmetry, thus twinning by reticular pseudopolyholohedry can be expected in jonesite: $\mathcal{T}_{\mathrm{T}} \subset \mathcal{T}_{\mathrm{I}}, \mathcal{D}(\mathbf{L})_{\mathrm{T}}=\mathcal{D}(\mathbf{L})_{\mathrm{I}}, \omega>0$. Actually, not all atoms refine anisotropically in the published structure, which is a possible consequence of undetected further twinning.

Twinning by (pseudo)polyholohedry has necessarily a relatively high twin index and, even if present, the crystal structure can in principle be solved because the overlapped nodes are a small fraction of the individual lattice $(1 / 5$ in the melilite case).

\section{Conclusions}

The revised classification of twinning in terms of the complete lattice symmetry has led to the introduction of a new category, reticular polyholohedry, and to establish a self-consistent scheme relating the various types of twinning.
The examples discussed, all taken from the recent literature, have shown the need to improve the geminographical knowledge and awareness of the daily crystallographer, who is confronted with twinned crystals and sometimes ends up with imprecise descriptions of his samples, even when he succeeds in solving and refining the structure. Often, daily crystallographers are interested more in the refinement of the molecular structure rather than in the crystallographic aspects of their samples. Nevertheless, a more geminographically aware approach to the treatment of twinned crystals would undoubtedly be of advantage for a clear approach to the structure solution of these crystals.

MN thanks Professor Elke Koch (Institute for Mineralogy, Petrology and Crystallography, University of Marburg, Germany) for profitable discussions. GF acknowledges the Italian Ministry of Education, University and Research (MIUR) for financial support. Dr Sergey V. Krivovichev (St Petersburg State University, Russia) kindly provided us with the data of jonesite before publication. We gratefully thank Professors Theo Hahn and Helmut Klapper (RWTH Aachen, Germany) for letting us know the content of Chapter 3.3 of Volume D of International Tables for Crystallography before its publication. The critical remarks by an anonymous referee were useful to improve the clearness of the manuscript.

\section{References}

Arakcheeva, A. V., Pushcharovskii, D. Yu., Gekimyants, V. M., Popov, V. A. \& Lubman, G. U. (1997). Crystallogr. Rep. 42, 54-63.

Bindi, L., Rees, H. \& Bonazzi, P. (2003). Acta Cryst. B59, 156-168. Buerger, M. J. (1945). Am. Mineral. 30, 469-482.

Buerger, M. J. (1954). An. Acad. Bras. Cien, 26, 111-121.

Buerger, M. J. (1962). X-ray Crystallography, pp. 53-67. New York: Wiley.

Catti, M. \& Ferraris, G. (1976). Acta Cryst. A32, 163-165.

Curien, H. \& Le Corre, Y. (1958). Bull. Soc. Fr. Minéral. Cristallogr. 81, 126-132.

Donnay, G. \& Donnay, J. D. H. (1974) Can. Mineral. 12, 422-425.

Ferraris, G., Gula, A., Ivaldi, G., Nespolo, M. \& Raade, G. (2001). Z. Kristallogr. 216, 442-448.

Ferraris, G., Makovicky, E. \& Merlino, S. (2004). Crystallography of Modular Materials. IUCr/Oxford University Press.

Ferraris, G. \& Nespolo, M. (2003). XXI European Crystallographic Congress, Program and Abstracts, p. 149.

Flippen-Anderson, J. L., Deschamps, J. R., Gilardi, R. D. \& George, C. (2001). Cryst. Eng. 4, 131-139.

Friedel, G. (1904). Étude sur les Groupements Cristallins. Extract from Bullettin de la Société de l'Industrie Minérale, Quatrième série, Tomes III et IV. Saint-Étienne: Societè de l'Imprimerie Thèolier J. Thomas et C.

Friedel, G. (1923). Bull. Soc. Fr. Minéral. 46, 79-95.

Friedel, G. (1926). Leçons de Cristallographie. Nancy/Paris/Strasbourg: Berger-Levrault.

Friese, K., Kienle, L., Duppel, V., Luo, H. \& Lin, C. (2003). Acta Cryst. B59, 182-189.

Giacovazzo, C. (2002a). Symmetry in Crystals. In Fundamentals of Crystallography, 2nd ed., edited by C. Giacovazzo. IUCr/Oxford University Press.

Giacovazzo, C. (2002b). Beyond Ideal Crystals. In Fundamentals of Crystallography, 2nd ed., edited by C. Giacovazzo. IUCr/Oxford University Press. 


\section{research papers}

Hahn, T. \& Klapper, H. (2003). Twinning of Crystals. International Tables for Crystallography, Vol. D, edited by A. Authier, Section 3.3. Dordrecht: Kluwer Academic Publishers.

Herbst-Irmer, R. \& Sheldrick, G. M. (1998). Acta Cryst. B54, 443-449.

Hodeau, J.-L., Tu, H. Y., Bordet, P., Fournier, T., Strobel, P., Marezio, M. \& Chandrashekhar, G. V. (1992). Acta Cryst. B48, 1-11.

Koch, E. (1992). Twinning. International Tables for Crystallography, Vol. C, edited by by A. C. J. Wilson, Section 1.3. Dordrecht: Kluwer Academic Publishers.

Krivovichev, S. V. \& Armbruster, Th. (2004). Am. Mineral. In the press.

Krivovichev, S. V., Yakovenchuk, V. N., Burns, P. C., Pakhomovsky, Y. A. \& Menshikov, Yu. P. (2003). Am. Mineral. 88, 424-429.

Mügge, O. (1898). N. Jahrb. Mineral. Geol. Palaeontol. 1, 71-158.

Nespolo, M. (2004). Z. Kristallogr. 219. In the press.

Nespolo, M. \& Ferraris, G. (2000). Z. Kristallogr. 215, 77-81.

Nespolo, M. \& Ferraris, G. (2003). Z. Kristallogr. 218, 178-181.
Quarez, E., Huve, M., Roussel, P. \& Mentré, O. (2002). J. Solid State Chem. 165, 214-227.

Shafranovskii, I. I. (1973). Lectures on Crystal Morphology. Washington: National Bureau of Standards of the USA.

Takano, Y. (1972). J. Jpn Assoc. Mineral. Petogr. Econ. Geol. 67, 345-351.

Takéuchi, Y (1997). Tropochemical Cell-Twinning. A Structure Building Mechanism in Crystalline Solids. Tokyo: Terra Scientific Publishing Company.

Tamazyan, R., Arnold, H., Molchanov, V., Kuzmicheva, G. \& Vasileva, I. (2000). Z. Kristallogr. 215, 346-351.

Wondratschek, H. (2002). Introduction to Space-Group Symmetry. International Tables for Crystallography, Vol. A, 5th ed., edited by Th. Hahn, Section 8, pp. 719-740. Dordrecht: Kluwer Academic Publishers.

Yeo, L., Harris, K. D. M. \& Kariuki, B. M. (2001). J. Solid State Chem. 156, 16-25. 